\title{
Protein crystal structures with ferrocene and ruthenocene-based enzyme inhibitors
}

\author{
Adam J. Salmon, ${ }^{a}$ Michael L. Williams, ${ }^{a}$ Andreas Hofmann ${ }^{a, b}$ and Sally-Ann Poulsen ${ }^{* a}$ \\ Received (in $X X X, X X X)$ Xth $X X X X X X X X X 20 X X$, Accepted Xth XXXXXXXXX 20XX \\ DOI: $10.1039 / b 000000 x$
}

We have determined the protein $X$-ray crystal structures of four organometallic inhibitors in complex with their target enzyme carbonic anhydrase II. The barrel shaped hydrophobic ferrocene and ruthenocene moieties have ${ }_{10}$ provided a structure-based avenue to better occupy the hydrophobic binding patch within the enzyme active site.

Protein X-ray structures of enzymes with bound inhibitors can reveal with intricate detail how the inhibitor interacts with the enzyme active site. There are however few reports in which an

15 organometallic inhibitor has been complexed with its target protein and a crystal structure obtained. Structures include a ruthenium-based half-sandwich complex with Pim-1 kinase (PDB ID 2BZH, 2BZI and 2BZJ), ${ }^{1}$ a ferrocene-based ligand with the antibody 13G5 (PDB ID 1A3L), ${ }^{2}$ and very recently a ruthenium ${ }_{20} d^{6}$-piano-stool complex with carbonic anhydrase II (PDB ID $3 \mathrm{PYK})^{3}$ and a half-sandwich ruthenium complex with glycogen synthase kinase $3 \beta$ (PDB ID 3M1S). ${ }^{4}$

Metallocenes are organometallic compounds comprising a sandwich structure wherein the metal ion is located between two 25 cyclopentadienyl $(\mathrm{Cp})$ rings. The $\pi$-electrons from each $\mathrm{Cp}$ ring are delocalised throughout the compound and this accounts for similar chemical behaviour of ferrocenes and ruthenocenes to that of aromatic compounds such as benzenes. The ferrocenyl moiety is air and water stable, non-toxic and its metabolism is 30 compatible with in vivo applications. ${ }^{5}$ There are numerous ferrocene-based compounds reported with good drug-like properties $^{5 \mathrm{a}}$ and several ferrocene-based compounds have found application in drug discovery, for example ferrocerone has been used to treat iron deficiency anaemia, ${ }^{6}$ while hydroxyferrocifen (a ${ }_{35}$ Tamoxifen $^{\mathrm{TM}}$ analogue) ${ }^{7}$ and ferroquine (a chloroquine analogue $)^{8}$ are in clinical development for the treatment of breast cancer and malaria, respectively. Ruthenocene and its derivatives are much less studied than their isostructural ferrocene counterparts, and to date only a small number of ruthenocene40 based compounds appear in the literature of drug discovery.

Carbonic anhydrases (CAs, EC 4.2.1.1) are zinc metalloenzymes that are validated therapeutic targets for a number of disease states including hypoxic tumours. ${ }^{9}$ CAs catalyse the reversible hydration of $\mathrm{CO}_{2}$ to generate a $\mathrm{HCO}_{3}{ }^{-}$and ${ }_{45} \mathrm{H}^{+} \cdot{ }^{9-10}$ Human CA II comprises a funnel shaped active site cavity at the base of which is a catalytic $\mathrm{Zn}^{2+}$ cation. This tetrahedral $\mathrm{Zn}^{2+}$ cation is coordinated to the imidazole sidechains of three histidine residues and to a $\mathrm{H}_{2} \mathrm{O}$ molecule, lowering the $\mathrm{p} K_{\mathrm{a}}$ of this water to $\sim 6.8$ (the $\mathrm{p} K_{\mathrm{a}}$ of bulk water is $\sim 14$ ) to facilitate formation
${ }_{50}$ of zinc bound hydroxide at physiological $\mathrm{pH} .{ }^{10}$ Hydroxide is the nucleophile that reacts with $\mathrm{CO}_{2}{ }^{10}$ Almost all reported small molecule CA inhibitors comprise a zinc binding group (ZBG) ${ }^{9}$ of which the primary sulfonamide group $\left(-\mathrm{SO}_{2} \mathrm{NH}_{2}\right)$ is recognised as the foremost example. The concept of the 'tail approach' for ${ }_{55}$ developing CA inhibitors was first described by Supuran and coworkers. ${ }^{11}$ 'Tail' moieties are linked to the primary sulfonamide ZBG to give an extended CA pharmacophore of [tail]-[aromatic][ZBG] and compounds that have a balanced physicochemical property profile needed for in vivo applications. ${ }^{12}$ We have ${ }_{60}$ previously reported the synthesis and CA inhibition of four metallocene-based CA inhibitors, compounds 1-4 (Fig. 1). ${ }^{13}$ Inhibitors 1-4 typify the [tail]-[aromatic]-[ZBG] pharmacophore, wherein the triazole-ferrocene or triazole-ruthenocene fragment comprises the 'tail' of the CA inhibitor. Inhibitors $\mathbf{1}$ and $\mathbf{3}$ are ${ }_{65}$ 1,4-disubstituted-1,2,3-triazoles synthesised by copper-catalysed azide-alkyne cycloaddition (CuAAC) of ethynyl ferrocene and ethynyl ruthenocene, respectively, and 4-azido benzenesulfonamide. Inhibitors $\mathbf{2}$ and $\mathbf{4}$ were synthesised from the same substrates using ruthenium-catalysed azide-alkyne 70 cycloaddition (RuAAC) and are the corresponding 1,5disubstituted-1,2,3-triazole regioisomers.

At hCA II ( $\mathrm{h}=$ human) the ferrocenyl 1,5-disubstituted triazole regioisomer $2\left(K_{\mathrm{i}}=36 \mathrm{nM}\right)$ has 2-fold better CA II inhibition than the 1,4-disubstituted triazole regioisomer $1\left(K_{\mathrm{i}}=80 \mathrm{nM}\right) .{ }^{13-14}$

75 The ruthenocene derivatives, with $K_{\mathrm{i}} \mathrm{s}$ of $9.7 \mathrm{nM}(3)$ and $12 \mathrm{nM}$ (4) nM, share similar hCA II inhibition and are 3- to 8-fold better hCA II inhibitors than their ferrocene counterparts. Compounds 1-4 are closely related in structure and likely to experience similar desolvation energies on binding to CA II. We therefore ${ }_{80}$ propose that the variable interactions of the barrel shaped hydrophobic metallocene moiety with the CA II protein may account for differences in inhibition to inform our understanding of structure-activity relationships and provide a structural base on which to direct future metallocene-based CA inhibitor design. To 85 obtain insight into the triazole-metallocene tail group interactions
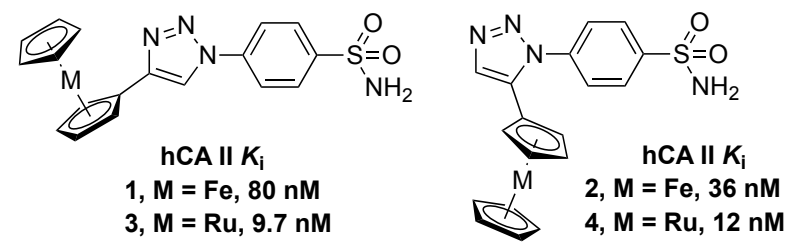

Fig. 1. Metallocene-based CA inhibitors 1 - 4. Human CA II inhibition constants $\left(K_{\mathrm{i}} \mathrm{s}\right)$ are shown. 
with the CA active site amino acids we have utilised protein $\mathrm{X}$ ray crystallography to determine the crystal structures of human CA II in complex with inhibitors 1-4. The crystal structures of the CA-metallocene-based inhibitor complexes were determined to 5 $2.0 \AA$ resolution (CA II:1), $1.5 \AA$ resolution (CA II:2), $2.2 \AA$ resolution (CA II:3) and $1.6 \AA$ resolution (CA II:4). Data collection and refinement statistics of inhibitor-bound CA II crystal structures are provided in ESI. ${ }^{\dagger}$ Coordinates and structure factors have been deposited with the PDB, with accession codes 10 3P55 (CA II:1), 3P3H (CA II:2), 3P44 (CA II:3) and 3P3J (CA II:4). The binding mode of the sulfonamide moiety to the catalytic zinc ion is invariant as compared to other sulfonamidebound CA II structures. The metallocene-triazole tail groups of the inhibitors occupy space in the active site cavity of CA II 15 without altering the configuration of residues lining the cavity. The binding cavity of CA comprises a cone-shaped active site with a hydrophobic and a hydrophilic face. ${ }^{10}$ The metallocenyl moieties are oriented towards the upper region of the hydrophobic face with both $\mathrm{Cp}$ rings involved in van der Waals 20 interactions with residues constituting the hydrophobic face (Fig. 2). Specifically, the metallocenyl moieties of the 1,4-regioisomers 1 and 3 engage in interactions Val134, Phe130, Pro201 and Leu203, while the metallocenyl moieties of the 1,5-regioisomers $\mathbf{2}$ and $\mathbf{4}$ are distant from Val134 and interact only with Phe130, ${ }_{25}$ Pro201 and Leu203. There are no direct interactions between protein residues and the central divalent ion of the metallocenyl groups. These observations are consistent with the only prior reported protein structure with a ferrocene-based ligand (PDB ID $1 \mathrm{~A} 3 \mathrm{~L}$ ) wherein several van der Waals contacts with the $\mathrm{Cp}$ rings ${ }_{30}$ are also observed. ${ }^{2}$ The shape of the ligands, as dictated by the regioisomeric relationship of the metallocenyl moieties, causes a tilt of the vertical axis of the benzenesulfonamide moiety of the 1,5-disubstituted-1,2,3-triazoles 2 and $\mathbf{4}$ compared to the 1,4disubstituted-1,2,3-triazoles 1 and 3 (Fig. 3). This tilt brings the

35 triazole of $\mathbf{2}$ and $\mathbf{4}$ into the vicinity of the active site lining residues and enables a hydrogen bond between $G \ln 192$ and this ligand. This hydrogen bond constitutes the key difference when comparing the binding interactions of $\mathbf{1 / 3}$ and $\mathbf{2} / \mathbf{4}$ with the protein (Fig. 2), and the 2-fold better CA II inhibition of $\mathbf{2}$ over $\mathbf{1}$ is 40 consistent with this additional hydrogen bond. In ligands $\mathbf{1}$ and $\mathbf{2}$, the $\mathrm{Cp}$ rings of the ferrocenyl moieties adopt a distorted staggered conformation offset with an angle close to $10^{\circ}$. The $\mathrm{Cp}$ rings of the ruthenocenyl compounds $\mathbf{3}$ and $\mathbf{4}$ are in eclipsed conformation. It has been shown that rotation of the two $\mathrm{Cp}$ rings 45 of ferrocene with respect to each other can occur in solution with a low energy barrier, ${ }^{15}$ and it has previously been observed that in the solid protein-bound state that the interactions between the $\mathrm{Cp}$ rings and their environment determines their relative orientation. ${ }^{2}$ The ferrocenyl ligand of PDB ID 1A3L has substituents on both ${ }_{50} \mathrm{Cp}$ rings that hydrogen bond to the antibody. $\mathrm{Cp}$ ring rotation appears restricted to maximize the number of specific interactions of the substituents with the antibody. The electron density provides no suggestion of multiple conformations, with the antibody acting to trap one conformer. ${ }^{2}$ In contrast, the

${ }_{55}$ unsubstituted $\mathrm{Cp}$ ring in compounds 1-4 of this study form only hydrophobic interactions with the protein residues, such that a relatively unrestricted rotation of the unsubstituted $\mathrm{Cp}$ ring with respect to the substituted $\mathrm{Cp}$ ring is likely. This assumption is supported by the electron density observed in the crystal ${ }_{60}$ structures of CA II:1-4 which is less well defined for the unsubstituted $\mathrm{Cp}$ ring than for the substituted $\mathrm{Cp}$ ring.

The backbone of the 1,4-disubstituted-1,2,3-triazoles 1 and $\mathbf{3}$ adopts a near-linear topology, whereas the 1,5-disubstituted1,2,3-triazoles $\mathbf{2}$ and $\mathbf{4}$ exhibit a corkscrew topology (Fig. 4), a ${ }_{65}$ phenomenon which has previously been observed with disubstituted 1,2,3-triazoles regioisomers. ${ }^{16}$ Both bound conformations are hypothesised to constitute the lowest energy conformations of the ligands in solution such that the binding of metallocenes 1-4 to human CA II does not require significant
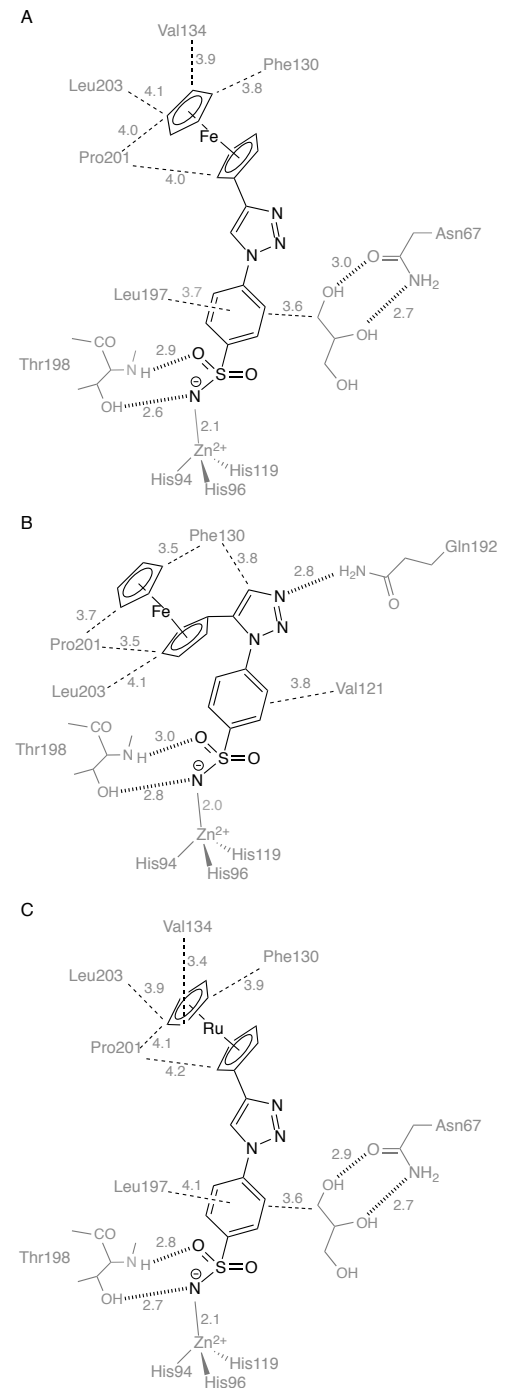

D

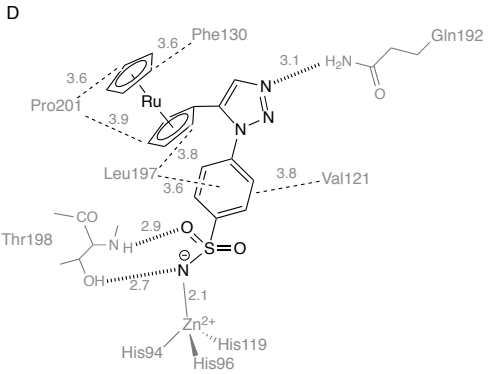

Figure 2. Protein-ligand interactions of metallocenes 1 (A), 2 (B), 3 (C) and 4 (D) in complex with human CA II. Hydrogen bonds - dotted lines, hydrophobic interactions - dashed lines. Atomic distances are in $\AA$. 
bond rotations to accommodate the ligands. The shape complementarity of the ligands in their most favourable conformations with parts of the CA II active site, combined with the stable anchoring of the compounds to the active site zinc 5 cation via the sulfonamide anion, are consistent with the good CA II inhibition constants observed. As well the ruthenium cation is larger than the iron cation and this increases the $\mathrm{Cp}$ ring separation in the ruthenocenyl ligands $\mathbf{3}$ and $\mathbf{4}$ compared to $\mathbf{1}$ and 2. This more subtle structural variation may account for the 10 observed difference in enzyme inhibition properties, with the ruthenocenyl ligands 3 and $\mathbf{4}$ 3- to 8-fold better CA II inhibitors than their ferrocenyl counterparts $\mathbf{1}$ and $\mathbf{2}$.

In conclusion, there are few reports in which an organometallic inhibitor has been complexed with its target protein and a crystal 15 structure obtained. Herein we have utilised protein X-ray crystallography to determine the crystal structures of human CA

20

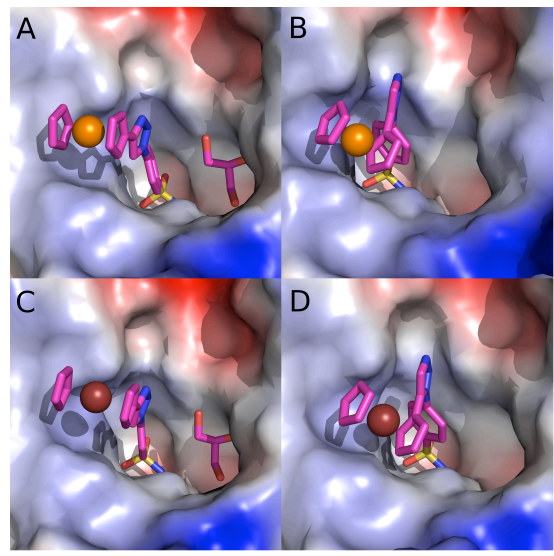

Fig. 3. Comparison of the space-filling in the active site of human CA II complexes with 1 (A), 2 (B), 3 (C) and 4 (D).
A

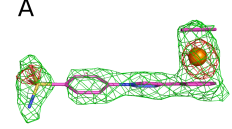

B

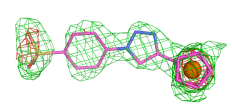

$\mathrm{E}$

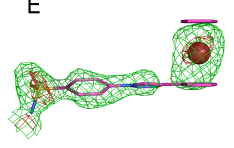

$\mathrm{F}$

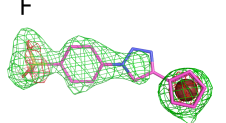

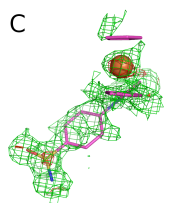

D

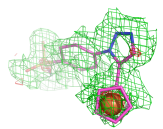

G

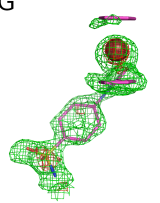

$\mathrm{H}$

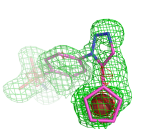

$30 \quad$ Fig. 4. Orthographic views depicting the linear $1(\mathbf{A}, \mathbf{B}), 3(\mathbf{E}, \mathbf{F})$ and corkscrew $2(\mathbf{C}, \mathbf{D}), \mathbf{4}(\mathbf{G}, \mathbf{H})$ geometry of metallocenes. The initial $\mathrm{F}_{\mathrm{o}}-\mathrm{F}_{\mathrm{c}}$ difference electron density (green) is contoured at $2 \sigma$. Anomalous density (red) is contoured at $2.5 \sigma$.
II in complex with two ferrocene- $(\mathbf{1}, \mathbf{2})$ and two ruthenocene35 based organometallic inhibitors $(3,4)$, to $1.5-2.2 \AA$ resolution. Structures (CAII:3) and (CAII:4) represent the first ruthenoceneprotein structures reported. While the metallocene moiety behaves chemically like an aromatic moiety such as a phenyl group, the barrel shaped sandwich structure of metallocene 40 permits access to 3D structural permutations that are not possible with a flat aromatic ring. This ligand attribute provides an enhanced opportunity to form a greater number of hydrophobic interactions with the hydrophobic face of the CA II binding cavity. Compounds also present two distinct ligand topologies 45 that impact on the shape complementarity towards the conical CA II active site cavity. In the context of protein-ligand interactions this may provide a discerning avenue to develop compounds to better occupy hydrophobic protein binding pockets within enzyme active sites. The implication of binding interactions 50 provided by these metallocene-based organometallic inhibitors has informed the on-going study of metallocene-based human CA inhibitors within our lab that have potential use as future drugs.

We thank the Australian Research Council (grant DP0877554 to S.-A.P), the Australian Government (Scholarship to A.J.S.) and ${ }_{55}$ the Australian Synchrotron (beam time to A.H.) for support.

\section{Notes and references}

${ }^{a}$ Eskitis Institute for Cell and Molecular Therapies, Griffith University, Nathan, Queensland 4111, Australia. Fax: 6173735 6001; Tel: 617 3735 7825; E-mail: s.poulsen@griffith.edu.au

$6{ }^{b}$ Faculty of Veterinary Science, The University of Melbourne, Victoria 3030, Australia

$\dagger$ Electronic Supplementary Information (ESI) available: Experimental section for protein X-ray crystallography. See DOI: 10.1039/b000000x/

1 J. É. Debreczeni, A. N. Bullock, G. E. Atilla, D. S. Williams, H.

65 Bregman, S. Knapp, E. Meggers, Angew. Chem. Int. Ed. 2006, 45, 1580 .

2 A. Heine, E. A. Stura, J. T. Yli-Kauhaluoma, C. Gao, Q. Deng, B. R. Beno, K. N. Houk, K. D. Janda, I. A. Wilson, Science 1998, 279, 1934.

703 F. W. Monnard, T. Heinisch, E. S. Nogueira, T. Schirmer, T. R. Ward, Chem. Commun. 2011, 47, 8238.

4 G. E. Atilla-Gokcumen, L. Di Costanzo, E. Meggers, J. Biol. Inorg. Chem. 2011, 16, 45

5 D. R. van Staveren, N. Metzler-Nolte, Chem. Rev. 2004, 104, 5931;

75 R. P. Hanzlik, P. Soine, W. H. Soine, J. Med. Chem. 1979, 22, 424; R. P. Hanzlik, W. H. Soine, J. Am. Chem. Soc. 1978, 100, 1290.

6 C. Imrie, L. Cook, D. C. Levendis, J. Organomet. Chem. 2001, 637639, 266.

7 S. Top, A. Vessieres, G. Leclercq, J. Quivy, J. Tang, J. Vaissermann,

$80 \quad$ M. Huche, G. Jaouen, Chem. Eur. J. 2003, 9, 5223.

8 D. Dive, C. Biot, ChemMedChem 2008, 3, 383.

9 C. T. Supuran, Nat. Rev. Drug Discov. 2008, 7, 168.

10 V. M. Krishnamurthy, G. K. Kaufman, A. R. Urbach, I. Gitlin, K. L. Gudiksen, D. B. Weibel, G. M. Whitesides, Chem. Rev. 2008, 108, 946.

11 A. Scozzafava, L. Menabuoni, F. Mincione, F. Briganti, G. Mincione, C. T. Supuran, J. Med. Chem. 1999, 42, 2641.

12 J.-Y. Winum, S.-A. Poulsen, C. Supuran, Med. Res. Rev. 2009, 29, 419.

9013 A. J. Salmon, M. L. Williams, A. Innocenti, D. Vullo, C. T. Supuran, S.-A. Poulsen, Bioorg. Med. Chem. Lett. 2007, 17, 5032.

14 M. Lopez, A. J. Salmon, C. T. Supuran, S.-A. Poulsen, Curr. Pharm. Des. 2010, 16, 3277.

15 J. T. Yli-Kauhaluoma, J. A. Ashley, C. Lo, L. Tucker, M. M. Wolfe, K. D. Janda, J. Am. Chem. Soc. 1995, 117, 7041.

16 Y. Bourne, H. Kolb, Z. Radic, K. Sharpless, P. Taylor, P. Marchot, PNAS 2004, 101, 1449. 